Bull. Austral. Math. Soc.

VOL. 59 (1999) [323-334]

\title{
QUELQUES REMARQUES SUR DES QUESTIONS D'APPROXIMATION DIOPHANTIENNE
}

\author{
PATRICE PHILIPPON
}

à Andreij Schinzel

Hoping for a hand-shake between methods from diophantine approximation theory

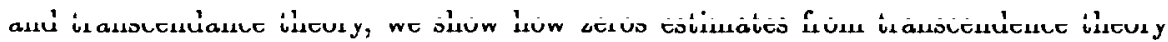
imply Roth's type lemmas (including the product theorem). We also formulate some strong conjectures on lower bounds for linear forms in logarithms of rational numbers with rational coefficients, inspired by the subspace theorem and which would imply, for example, the $a b c$ conjecture.

Nous montrons dans cette note quelques faits qui semblent indiquer une possible conciliation entre les méthodes développées dans le cadre du théorème du sous-espace (RothSchmidt, see [9]) d'une part et dans le cadre des minorations de formes linéaires de logarithmes (méthode de Baker) d'autre part ... pour le plus grand bien de l'approximation diophantienne.

Dans un premier temps (Sections 1 et 2) nous montrons comment le lemme de Roth et ses récentes généralisations (dont le théorème du produit, voir $[2,4]$ ) se déduisent naturellement du lemme de zéros dans les groupes algébriques de [7], appliqué aux puissances du groupe additif $\mathbf{G}_{a}$. Ceci éclaire le parallèle mis en évidence dans [6] et permet d'obtenir de petits raffinements quantitatifs des théorèmes de [2]. Rappelons que le lemme de zéros de [7] est un outil ordinaire des minorations de formes linéaires de logarithmes.

Ensuite, nous formulons (Section 3) des conjectures sur les minorations de formes linéaires de logarithmes de nombres rationnels à coefficients rationnels, s'inspirant des conjectures les plus optimistes dans ce domaine et de la formulation du théorème du sous-espace de Schlickewei [8]. Nous montrons comment notre conjecture la plus forte entraîne simplement la conjecture $a b c$.

Received 30th September, 1998

Je remercie les collègues dont l'interet pour ce travail m'a permis d'en améliorer grandement la forme et le fond.

Copyright Clearance Centre, Inc. Serial-fee code: 0004-9729/99 \$A2.00+0.00. 


\section{LEMME DE ROTH}

Soient $g \in \mathbf{N}^{*}, \underline{\delta}=\left(\delta_{1}, \ldots, \delta_{g}\right) \in \mathbf{N}^{g}$, nous notons

$$
A_{g}=\overline{\mathbf{Q}}\left[X_{0,1}, X_{1,1}, \ldots, X_{0, g}, X_{1, g}\right]
$$

et, pour $\varepsilon \in \mathbf{R}_{>0}, W(\underline{\delta}, \varepsilon)=\left\{\alpha \in \mathbf{N}^{g} ;\left(\alpha_{1} / \delta_{1}\right)+\cdots+\left(\alpha_{g} / \delta_{g}\right)<\varepsilon\right\}$. Nous considérons $\overline{\mathbf{Q}}^{g}$ comme les points définis sur $\overline{\mathbf{Q}}$ du groupe additif $\mathbf{G}_{a}^{g}$ plongé dans $\mathrm{P}_{1}^{g}$ et nous identifions de la façon naturelle l'espace tangent en un point de $\mathbf{G}_{a}^{g}(\mathbf{C})$ à $\mathbf{C}^{g}$. Soit $x \in \mathbf{G}_{a}^{g}(\overline{\mathbf{Q}})$, suivant la terminologie de [7] nous dirons que $\Sigma(\underline{\delta}, \varepsilon):=W(\underline{\delta}, \varepsilon) \times\{x\}$ est un ensemble pondéré, l'indice $i_{\underline{\delta}}(P, x)$ d'une forme multihomogène $P \in A$ en $x$, utilisé dans [2] et [4], est alors l'infimum des $\varepsilon \in \mathbf{R}_{>0}$ tels que $P$ s'annule sur l'ensemble pondéré $\Sigma(\underline{\delta}, \varepsilon)$ (au sens de [7]). Nous notons d'une même lettre $h$ la hauteur logarithmique, absolue d'un élément de $\overline{\mathbf{Q}}$ et la hauteur (logarithmique, absolue) d'un polynôme à coefficients dans $\overline{\mathbf{Q}}$ définie à l'aide de la mesure de Mahler pour les places infinies.

THÉREME 1. Soient $\varepsilon \in \mathbf{R}_{>0}, g \in \mathbf{N}^{*}, x=\left(x_{1}, \ldots, x_{g}\right) \in \mathbf{G}_{a}^{g}(\overline{\mathbf{Q}}), \underline{\delta}=$ $\left(\delta_{1}, \ldots, \delta_{g}\right) \in \mathrm{N}^{g}$ et $P \in A_{g}$ une forme non nulle de multidegré $\underline{\delta}$. On suppose

$$
\begin{gathered}
\delta_{i} / \delta_{i+1}>g / \varepsilon \geqslant 1, \quad i=1, \ldots, g-1, \quad \text { et } \\
h(P)+\delta_{1}+\cdots+\delta_{g} \leqslant \min _{1 \leqslant i \leqslant g}\left(\delta_{i}(\varepsilon / g+1)^{g} h\left(x_{i}\right)\right),
\end{gathered}
$$

alors la forme $P$ ne s'annule pas sur l'ensemble pondéré $\Sigma(\underline{\delta}, g \varepsilon)$ (c'est-à-dire, $i_{\underline{\delta}}(P, x) \leqslant$ $g \varepsilon)$.

DÉMONSTRation. On raisonne par l'absurde, posons $W=W(\underline{\delta}, \varepsilon)$, le Théorème 2 de [7] (et la remarque subséquente sur les hauteurs [Comme me l'a fait remarqué G. Rémond, cette remarque n'indique pas correctement les modifications à apporter à la preuve du lemme 5 de [7] pour inclure l'estimation des hauteurs. Il convient en fait d'en donner une preuve différente basée sur la construction d'une suite régulière maximale dans $I A_{\mathfrak{p}}$, plutôt que d'une suite superficielle.]) appliqué avec $E=\{x\}, \Sigma_{0}=\{(0,0)\}$ et $\Sigma_{1}=\cdots=$ $\Sigma_{g}=W \times\{0\}$ entraîne que, si $P$ s'annule sur $\Sigma(\underline{\delta}, g \varepsilon)$ alors il existe une sous-variété algébrique propre $V$ de $\mathbf{P}_{1}^{g}$ de dimension $d$, contenant $x$ et satisfaisant

$$
\begin{gathered}
m_{W}(V) \cdot d ! \sum_{\substack{\ell \in\{0,1\}^{g} \\
|\ell|=d}} d_{\ell}(V) \delta_{1}^{\ell_{1}} \ldots \delta_{g}^{\ell_{g}} \leqslant g ! \delta_{1} \ldots \delta_{g} \\
m_{W}(V) .(d+1) ! \sum_{\substack{\ell \in\{0,1\}^{g} \\
|\ell|=d+1}} h_{\ell}(V) \delta_{1}^{\ell_{1}} \ldots \delta_{g}^{\ell_{g}} \leqslant(g+1) !\left(h(P)+\delta_{1}+\cdots+\delta_{g}\right) \delta_{1} \ldots \delta_{g},
\end{gathered}
$$

où $d_{\ell}(V), h_{\ell}(V)$ désignent les degrés et hauteurs multi-projectives de $V$ et $m_{W}(V)=$ $\varepsilon^{g-d} \max \left(\delta_{1}^{\ell_{1}} \ldots \delta_{g}^{\ell_{g}}\right)$, le maximum étant pris sur les $\ell \in\{0,1\}^{g},|\ell|=g-d$ tels que le sous-espace de $\mathrm{C}^{g}$ engendré par les facteurs correspondants aux $\ell_{\alpha} \neq 0$ se projette 
surjectivement sur $\mathrm{C}^{g}$ modulo $T_{y} V$, l'espace tangent à $V$ en un point général $y \in V$. Nous allons en tirer une contradiction avec les conditions (1) et (2) en distinguant deux cas. On remarque que si $d_{i}(V) \neq 0$ alors le sous-espace de $\mathbf{C}^{g}$ engendré par les facteurs d'indice $\bar{\imath}$ tel que $\bar{\imath}_{\alpha}=1-i_{\alpha}$, se projette surjectivement sur $\mathbf{C}^{g}$ modulo $T_{y} V$. On peut également supposer qu'aucun $\mathbf{P}_{1}$ n'est facteur de $V$ car si $V=\mathbf{P}_{1} \times V_{1}\left(\mathbf{P}_{1}\right.$ étant le $\alpha$-ième facteur de $\left.\mathbf{P}_{1}^{g}\right)$ et $i \in\{0,1\}^{g}, i_{\alpha}=0$, on a $d_{i}\left(V_{1}\right)=d_{i^{\prime}}(V)$ où $i_{\beta}^{\prime}=i_{\beta}$ pour $\beta \neq \alpha$ et $i_{\alpha}^{\prime}=1$, puis une égalité analogue pour les hauteurs et enfin $m_{W}\left(V_{1}\right)=m_{W}(V)$. On désigne par $\pi_{\alpha}$ la projection sur le $\alpha$-ième facteur de $\mathrm{P}_{1}^{g}$.

PrEmier CAS. Il existe $1 \leqslant \alpha \leqslant g$ tel que $\operatorname{dim} \pi_{\alpha}(V)=0$. Dans ce cas on peut considérer des multi-indices $i, i^{\prime},|i|=d, i_{\beta}^{\prime}=i_{\beta}$ pour $\beta \neq \alpha, i_{\alpha}^{\prime}=1, i_{\alpha}=0$, tels que $d_{i}(V) \neq 0$, on a $h_{i^{\prime}}(V)=h\left(\pi_{\alpha}(V)\right) \cdot d_{i}(V)$. Le sous-espace de $C^{y}$ engendre par les tacteurs d'ináice $\imath$ se projette surjectivement sur $\mathbf{C}^{g}$ modulo $T_{y} V$ et on a $m_{W}(V) \geqslant \varepsilon^{g-d} \cdot\left(\delta_{1} \ldots \delta_{g}\right) /\left(\delta_{1}^{i_{1}} \ldots \delta_{g}^{i_{g}}\right)$. On écrit

$$
m_{W}(V) \cdot(d+1) ! \sum_{\substack{\ell \in\{0,1\}^{g} \\|\ell|=d+1}} h_{\ell}(V) \delta_{1}^{\ell_{1}} \ldots \delta_{g}^{\ell_{g}} \geqslant(d+1) ! \varepsilon^{g-d} h\left(x_{\alpha}\right) \delta_{\alpha} \delta_{1} \ldots \delta_{g},
$$

$\operatorname{car} h_{i^{\prime}}(V) \geqslant h\left(x_{\alpha}\right)$. Comparant à la majoration ci-dessus il vient

$$
\left(\frac{\varepsilon}{g+1}\right)^{g-d} h\left(x_{\alpha}\right) \delta_{\alpha} \leqslant \frac{\varepsilon^{g-d}}{(d+2) \ldots(g+1)} \cdot h\left(x_{\alpha}\right) \delta_{\alpha} \leqslant h(P)+\delta_{1}+\cdots+\delta_{g}
$$

en contradiction avec (2), car $\varepsilon \leqslant g$.

SECOND CAS. Pour tout $1 \leqslant \alpha \leqslant g$ on a $\pi_{\alpha}(V)=\mathbf{P}_{1}$. Dans ce cas on a en particulier $d \geqslant 1$ et il existe des multi-indices $i, i^{\prime},|i|=\left|i^{\prime}\right|=d, i_{\alpha}=i_{\alpha}^{\prime}$ pour $1<\alpha<g$, $i_{1}=i_{g}^{\prime}=1, i_{g}=i_{1}^{\prime}=0$, tels que $d_{i}(V) \neq 0$ et $d_{i^{\prime}}(V) \neq 0$. Le sous-espace de $\mathrm{C}^{g}$ engendré par les facteurs d'indice $\bar{\imath}^{\prime}$ se projette surjectivement sur $\mathrm{C}^{g}$ modulo $T_{y} V$, on a $m_{W}(V) \geqslant \varepsilon^{g-d} .\left(\delta_{1} \ldots \delta_{g}\right) /\left(\delta_{1}^{i_{1}^{\prime}} \ldots \delta_{g}^{i_{g}^{\prime}}\right)$ et on peut donc écrire

$$
m_{W}(V) . d ! \sum_{\substack{\ell \in\{0,1\}^{g} \\|\ell|=d}} d_{\ell}(V) \delta_{1}^{\ell_{1}} \ldots \delta_{g}^{\ell_{g}} \geqslant d ! m_{W}(V) \delta_{1}^{i_{1}} \ldots \delta_{g}^{i_{g}} \geqslant d ! \varepsilon^{g-d} \delta_{1} \ldots \delta_{g} \cdot \frac{\delta_{1}}{\delta_{g}}
$$

Comparant à la majoration ci-dessus il vient

$$
\frac{\delta_{1}}{\delta_{g}}=\prod_{i=1}^{g-1} \frac{\delta_{i}}{\delta_{i+1}} \leqslant \frac{g !}{d !} \cdot \varepsilon^{d-g} \leqslant\left(\frac{g}{\varepsilon}\right)^{g-d}
$$

en contradiction avec (1), $\operatorname{car} \prod_{i=1}^{g-1}\left(\delta_{i} / \delta_{i+1}\right)>(g / \varepsilon)^{g-1} \geqslant(g / \varepsilon)^{g-d}$.

On obtient ainsi une contradiction en tout cas, ce qui établit l'énoncé du théorème. [ 
L'argument de la démonstration ci-dessus est d'injecter la condition (1) dans la conclusion du lemme de zéros de [7] afin de la préciser. Nous développons cette idée au paragraphe suivant pour retrouver et raffiner diverses généralisations du lemme de Roth. Notons en passant que c'est la finesse des énoncés de [7] qui permet de faire le lien avec le lemme de Roth et ses généralisations (lorsqu'on les applique à $\mathbf{G}_{a}^{g}$ ), les lemmes de zéros sur les groupes algébriques commutatifs antérieurs ne donneraient que des versions faibles de ces résultats.

\section{GÉnÉRAlisations du lemme de Roth}

Nous donnons une généralisation du lemme de Roth en considérant $\overline{\mathbf{Q}}^{n}$ comme les points définis sur $\overline{\mathbf{Q}}$ du groupe additif $\mathbf{G}_{a}^{n}$ plongé dans $\mathbf{P}:=\mathbf{P}_{n_{1}} \times \cdots \times \mathbf{P}_{n_{g}}$ où $n=$ $n_{1}+\cdots+n_{g}$. Soit $K$ un corps de nombres, nous posons maintenant

$$
A=A(\mathbf{P})=K\left[X_{0,1}, \ldots, X_{n_{1}, 1}, \ldots, X_{0, g}, \ldots, X_{n_{g}, g}\right]
$$

et, pour $\underline{\delta}=\left(\delta_{1}, \ldots, \delta_{g}\right) \in \mathrm{N}^{g}, \varepsilon>0$,

$$
W(\underline{\delta}, \varepsilon)=\left\{\left(\alpha_{1}, \ldots, \alpha_{g}\right) \in \mathbf{N}^{n_{1}} \times \cdots \times \mathbf{N}^{n_{g}} ; \frac{\left|\alpha_{1}\right|}{\delta_{1}}+\cdots+\frac{\left|\alpha_{g}\right|}{\delta_{g}}<\varepsilon\right\},
$$

où pour $\alpha \in \mathrm{N}^{s}$ on a noté $|\alpha|$ la somme des coordonnées de $\alpha$. Soit $E$ une sous-variété algébrique de $\mathrm{P}$, on considère l'ensemble pondéré $\Sigma(\underline{\delta}, \varepsilon):=W(\underline{\delta}, \varepsilon) \times E$ et l'indice $i_{\underline{\delta}}(P, E)$ d'une forme multihomogène $P \in A$ est l'infimum des $\varepsilon \in \mathbf{R}_{>0}$ tels que $P$ s'annule sur l'ensemble pondéré $\Sigma(\underline{\delta}, \varepsilon)$ (au sens de [7]), on a $i_{\underline{\delta}}(P, E)=\inf _{x \in E} i_{\underline{\delta}}(P, x)$. On peut alors énoncer:

ThÉoREme 2. Soient $\varepsilon \in \mathbf{R}_{>0}, g \in \mathbf{N}^{*}, n_{1}, \ldots, n_{g} \in \mathbf{N}^{*}, E$ une sous-variété algébrique de $\mathbf{P}, \underline{\delta}=\left(\delta_{1}, \ldots, \delta_{g}\right) \in \mathbb{N}^{g}$ et $P \in A$ une forme de multidegré $\underline{\delta}$. On pose $n=n_{1}+\cdots+n_{g}$, on suppose $0<\varepsilon \leqslant 1$ et

$$
\delta_{i} / \delta_{i+1}>(g / \varepsilon)^{n}, \quad i=1, \ldots, g-1,
$$

alors, si $P$ s'annule sur l'ensemble pondéré $\Sigma(\underline{\delta}, n \varepsilon)$ (c'est-à-dire, $i_{\underline{\delta}}(P, x)>n \varepsilon$ en tout point $x \in E$ ) il existe une sous-variété $V$ de $\mathbf{P}$ définie sur $K$, contenant $E$, dont toutes les composantes irréductibles sur $\bar{K}$ sont des sous-variétés produits de $\mathbf{P}$, on a $V=$ $\bigcup_{\sigma \in \operatorname{Gal}(\bar{K} / K)} \sigma(\tilde{V})$ où $\tilde{V}=V_{1} \times \cdots \times V_{g}$ et en posant $D:=d(V) / d(\tilde{V})$ :

$$
\begin{gathered}
D . d\left(V_{1}\right) \ldots d\left(V_{g}\right) \leqslant\left(\frac{n}{\varepsilon}\right)^{n-\operatorname{dim} V}, \\
D . d\left(V_{1}\right) \ldots d\left(V_{g}\right) \sum_{i=1}^{g} \delta_{i} \frac{h\left(V_{i}\right)}{d\left(V_{i}\right)} \leqslant\left(\frac{n+1}{\varepsilon}\right)^{n-\operatorname{dim} V} \cdot\left(h(P)+\sum_{i=1}^{g} \delta_{i} \log \left(n_{i}+1\right)\right) .
\end{gathered}
$$

De plus, $i_{\underline{\delta}}(P, x)>\varepsilon$ en tout point $x \in V$. 
DÉmonstration. Posons $W=W(\underline{\delta}, \varepsilon)$, le Théorème 2 de [7] (et la remarque subséquente sur les hauteurs ) appliqué avec $E, \Sigma_{0}=\{(0,0)\}$ et $\Sigma_{1}=\cdots=\Sigma_{g}=W \times\{0\}$ entraîne que, $P$ s'annulant sur $\Sigma(\underline{\delta}, n \varepsilon)$, il existe une sous-variété algébrique propre $V$ de $\mathrm{P}$ définie sur $K$, de dimension $d$, contenant $E$ et satisfaisant

$$
\begin{gathered}
m_{W}(V) \cdot H_{g}(V ; \underline{\delta}) \leqslant\left(\begin{array}{c}
n \\
n_{1} \ldots n_{g}
\end{array}\right) \cdot \delta_{1}^{n_{1}} \ldots \delta_{g}^{n_{g}} \\
m_{W}(V) \cdot H_{a}(V ; \underline{\delta}) \leqslant(n+1) \cdot\left(\begin{array}{c}
n \\
n_{1} \ldots n_{g}
\end{array}\right) \cdot\left(h(P)+\sum_{i=1}^{g} \delta_{i} \log \left(n_{i}+1\right)\right) \delta_{1}^{n_{1}} \ldots \delta_{g}^{n_{g}},
\end{gathered}
$$

où $H_{g}(V ; \underline{\delta})$ et $H_{a}(V ; \underline{\delta})$ désignent les formes degré et hauteur multi-projectives de $V$ en

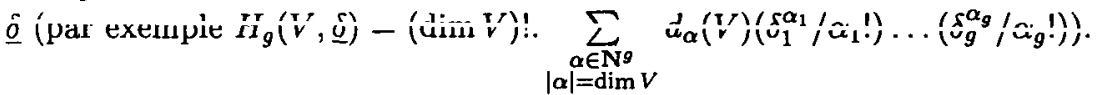

Soit $\widetilde{V}$ une composante irréductible sur $\bar{K}$ de $V, \pi_{i}$ la projection de $\mathbf{P}$ sur le facteur $\mathbf{P}_{n_{i}}, x_{i}$ un point suffisamment général de $\pi_{i}(\tilde{V})$ et

$$
W_{i}=\tilde{V} \cap\left(\left\{x_{1}\right\} \times \cdots \times\left\{x_{i-1}\right\} \times \mathbf{P}_{n_{i}} \times\left\{x_{i+1}\right\} \times \cdots \times\left\{x_{g}\right\}\right),
$$

si $\widetilde{V}$ n'est pas produit de ses projections sur les différents $\mathbf{P}_{n_{i}}$ il existe $1 \leqslant i<g$ tel que $\operatorname{dim} \pi_{i}(\tilde{V})>\operatorname{dim} W_{i}$. On choisit $i$ minimal avec cette propriété et, pour $j=1, \ldots, i$ on pose $\alpha_{j}=\operatorname{dim} \pi_{j}(V)$ et $\beta_{j}=n_{j}-\operatorname{dim} W_{j}$, on a $\alpha_{i}+\beta_{i}>n_{i}$. On complète en des indices $\alpha$ et $\beta$ dans $\mathrm{N}^{g}$ de sorte qu'il soient minimaux pour l'ordre lexicographique satisfaisant $d_{\alpha}(V) \neq 0$ et $m_{W}(V) \geqslant \delta_{1}^{\beta_{1}} \ldots \delta_{g}^{\beta_{g}} \varepsilon^{|\beta|}$. Ceci est clairement possible grâce à la propriété de minimalité de $i$. Comme $|\alpha|=\operatorname{dim} V,|\beta|=n-\operatorname{dim} V$ et $\delta_{1}^{\alpha_{1}+\beta_{1}} \ldots \delta_{g}^{\alpha_{g}+\beta_{g}} \geqslant$ $\left(\delta_{i} / \delta_{j}\right) . \delta_{1}^{n_{1}} \ldots \delta_{g}^{n_{g}}$ pour un certain indice $j>i$, on en déduit

$$
\frac{\delta_{i}}{\delta_{i+1}} \cdot \varepsilon^{n-\operatorname{dim} V} \cdot \delta_{1}^{n_{1}} \ldots \delta_{g}^{n_{g}} \leqslant m_{W}(V) \cdot H_{g}(V ; \underline{\delta}) \leqslant\left(\begin{array}{c}
n \\
n_{1} \ldots n_{g}
\end{array}\right) \cdot \delta_{1}^{n_{1}} \ldots \delta_{g}^{n_{g}}
$$

d'où $\delta_{i} / \delta_{i+1} \leqslant(g / \varepsilon)^{n}$ en contradiction avec (3). Ainsi $\widetilde{V}=V_{1} \times \cdots \times V_{g}$ est bien un produit de variétés et les majorations des degré et hauteur résultent de celles ci-dessus car alors

$$
\begin{aligned}
m_{W}(V) & \geqslant \varepsilon^{n-\operatorname{dim} V} \cdot \delta_{1}^{n_{1}-\operatorname{dim} V_{1}} \ldots \delta_{g}^{n_{g}-\operatorname{dim} V_{g}} \\
H_{g}(V ; \underline{\delta}) & =D \cdot\left(\begin{array}{c}
\operatorname{dim} V \\
\operatorname{dim} V_{1} \ldots \operatorname{dim} V_{g}
\end{array}\right) \cdot d\left(V_{1}\right) \ldots d\left(V_{g}\right) \delta_{1}^{\operatorname{dim} V_{1}} \ldots \delta_{g}^{\operatorname{dim} V_{g}} \\
H_{a}(V ; \underline{\delta}) & =H_{g}(V ; \underline{\delta}) \cdot \sum_{i=1}^{g} \frac{\operatorname{dim} V+1}{\operatorname{dim} V_{i}+1} \cdot \delta_{i} \cdot \frac{h\left(V_{i}\right)}{d\left(V_{i}\right)}
\end{aligned}
$$

et on peut supposer $\operatorname{dim} V_{i}+1 \leqslant n_{i}$ pour $i=1, \ldots, g$.

Corolla ire 3. Dans les notations et hypothèses du Théorème 2 on a: 
(i) si $E$ est une composante irréductible sur $\bar{K}$ de $\left\{x ; i_{\underline{\delta}}(P, x)>\varepsilon\right\}$ alors $E=\widetilde{V}$ est une variété produit dans $\mathbf{P}$;

(ii) le stabilisateur de $V$ dans $\mathrm{G}_{a}^{n}$ (c'est-à-dire, $\left\{x \in \mathrm{G}_{a}^{n}(\bar{K}) ; x+V=V\right\}$ ) est un produit de sous-espaces linéaires $L=L_{1} \times \cdots \times L_{g}$ et $i_{\underline{\delta}}(P, x)>\varepsilon$ en tout point $x \in E+L$;

(iii) si $E=E_{1} \times \cdots \times E_{g}$ où $E_{i}$ est un hyperplan de $\mathbf{P}_{n_{i}}$, alors il existe $1 \leqslant i \leqslant g$ tel que $\delta_{i} \varepsilon^{g} h\left(E_{i}\right) \leqslant(n+1)^{g} \cdot\left(h(P)+\sum_{i=1}^{g} \delta_{i} \log \left(n_{i}+1\right)\right)$.

DÉmonstration. La première assertion est claire et la seconde suit de $E+L \subset V$. Pour la troisième on remarque qu'un des $V_{i}$ au moins est de codimension 1 et donc $V_{i}=E_{i}$. La conclusion suit de la dernière majoration du théorème, car $n-\operatorname{dim} V \leqslant g$.

L'énoncé (i) du corollaire correspond à la situation du théorème du produit de Faltings [3], et les estimations du Théorème 2 améliorent un peu les versions effectives de ce résultat, établies dans [2] et [4] .

Lorsque $n_{1}=\cdots=n_{g}=1$, la conclusion (iii) du Corollaire (3) est la négation de (2) du Théorème 1 , mais on ne retrouve pas ce théorème comme corollaire du Théorème 2 car la condition (3) est plus forte que (1). Une généralisation du théorème de Roth s'énonce comme suit.

THÉORÈme 4. Soient $\varepsilon \in \mathbf{R}_{>0}, g \in \mathbf{N}^{*}, n_{1}, \ldots, n_{g} \in \mathbf{N}^{*}, E=E_{1} \times \cdots \times E_{g}$ un produit d'hyperplans dans $\mathbf{P}, \underline{\delta}=\left(\delta_{1}, \ldots, \delta_{g}\right) \in \mathrm{N}^{g}$ et $P \in A$ une forme de multidegré $\underline{\delta}$. On pose $n=n_{1}+\cdots+n_{g}$ et on suppose

$$
\begin{gathered}
\delta_{i} / \delta_{i+1}>n / \varepsilon \geqslant 1, \quad i=1, \ldots, g-1 \quad \text { et } \\
h(P)+\sum_{i=1}^{g} \delta_{i} \log \left(n_{i}+1\right) \leqslant \min _{1 \leqslant i \leqslant g}\left(\delta_{i}(\varepsilon / n+1)^{g} h\left(E_{i}\right)\right),
\end{gathered}
$$

alors la forme $P$ ne s'annule pas sur l'ensemble pondéré $\Sigma(\underline{\delta}, n \varepsilon)$ (c'est-à-dire, $i_{\underline{\delta}}(P, x) \leqslant$ $n \varepsilon$ pour au moins un $x \in E$ ).

DÉMONSTRATION. Comme dans la démonstration du Théorème 1 on raisonne par l'absurde, on peut supposer qu'aucun $\mathbf{P}_{n_{i}}$ n'est facteur de $V$ et on distingue deux cas. On reprend les notations du début de la démonstration du Théorème 2 en supposant que $P$ s'annule sur $\Sigma(\underline{\delta}, n \varepsilon)$, en particulier $\operatorname{dim} V \geqslant n-g$ car $V \supset E$.

Premier CAS. Il existe $1 \leqslant \alpha \leqslant g$ tel que $\operatorname{dim} \pi_{\alpha}(V)=n_{\alpha}-1$, on a $\pi_{\alpha}(V)=E_{\alpha}$. Dans ce cas on peut considérer des multi-indices $i, i^{\prime},|i|=\operatorname{dim} V, i_{\beta}^{\prime}=i_{\beta}$ pour $\beta \neq \alpha$, $i_{\alpha}=n_{\alpha}-1$ et $i_{\alpha}^{\prime}=n_{\alpha}$, tels que $d_{i}(V) \neq 0$. Le sous-espace engendré par les facteurs de $\mathbf{C}^{g}$ d'indice $\bar{\imath}$ tel que $\bar{\imath}_{\beta}=n_{\beta}-i_{\beta}$, se projette surjectivement sur $\mathbf{C}^{g}$ modulo $T_{y} V$ et on a $m_{W}(V) \geqslant \varepsilon^{n-\operatorname{dim} V} \cdot\left(\delta_{1}^{n_{1}} \ldots \delta_{g}^{n_{g}}\right) /\left(\delta_{1}^{i_{1}} \ldots \delta_{g}^{i_{g}}\right)$. On écrit

$$
m_{W}(V) \cdot H_{a}(V ; \underline{\delta}) \geqslant \frac{(\operatorname{dim} V+1) !}{i_{1}^{\prime} ! \ldots i_{g}^{\prime} !} \cdot \varepsilon^{n-\operatorname{dim} V} h\left(E_{\alpha}\right) \delta_{\alpha} \delta_{1}^{n_{1}} \ldots \delta_{g}^{n_{g}}
$$


car $h_{i^{\prime}}(V)=h\left(E_{\alpha}\right) \cdot d_{i}(V) \geqslant h\left(E_{\alpha}\right)$. Comparant à la majoration du début de la preuve du Théorème 2 il vient

$$
\left(\frac{\varepsilon}{n+1}\right)^{n-\operatorname{dim} V} h\left(E_{\alpha}\right) \delta_{\alpha} \leqslant h(P)+\sum_{i=1}^{g} \delta_{i} \log \left(n_{i}+1\right)
$$

en contradiction avec (5), $\operatorname{car} \varepsilon \leqslant n$ et $n-\operatorname{dim} V \leqslant g$.

SECOND CAS. Pour tout $1 \leqslant \alpha \leqslant g$ on a $\pi_{\alpha}(V)=\mathbf{P}_{n_{\alpha}}$. Dans ce cas on a $\operatorname{dim} V \geqslant n-g+1$ et il existe des multi-indices $i, i^{\prime},|i|=\left|i^{\prime}\right|=\operatorname{dim} V, i_{\alpha}=i_{\alpha}^{\prime}$ pour $1<\alpha<g, i_{1}=n_{1}$, $i_{1}^{\prime}=n_{1}-1, i_{g}=n_{g}-1$ et $i_{g}^{\prime}=n_{g}$, tels que $d_{i}(V) \neq 0$ et $d_{i^{\prime}}(V) \neq 0$. Le sous-espace engendré par les facteurs de $\mathbf{C}^{g}$ d'indice $\vec{\imath}^{\prime}$ se projette surjectivement sur $\mathbf{C}^{g}$ modulo $T_{y} V$. On peut donc écrire

$$
m_{W}(V) \cdot H_{g}(V ; \underline{\delta}) \geqslant \frac{\operatorname{dim} V !}{i_{1} ! \ldots i_{g} !} \cdot \varepsilon^{n-\operatorname{dim} V} \delta_{1}^{n_{1}} \ldots \delta_{g}^{n_{g}} \cdot \frac{\delta_{1}}{\delta_{g}} .
$$

Comparant à la majoration du début de la preuve du Théorème 2 il vient

$$
\frac{\delta_{1}}{\delta_{g}}=\prod_{i=1}^{g-1} \frac{\delta_{i}}{\delta_{i+1}} \leqslant \frac{n !}{\operatorname{dim} V !} \cdot \varepsilon^{\operatorname{dim} V-n} \leqslant\left(\frac{n}{\varepsilon}\right)^{n-\operatorname{dim} V}
$$

en contradiction avec (4), $\operatorname{car} \prod_{i=1}^{g-1}\left(\delta_{i} / \delta_{i+1}\right)>(n / \varepsilon)^{g-1} \geqslant(n / \varepsilon)^{n-\operatorname{dim} V}$.

On obtient ainsi une contradiction en tout cas, ce qui établit l'énoncé du théorème.

\section{Conjectures.}

En regard des améliorations possibles, le théorème du sous-espace énumératif semble beaucoup plus proche de l'optimal que les minorations de formes linéaires de logarithmes. Nous voulons maintenant discuter une conjecture sur ces dernières et ses conséquences pour la conjecture $a b c$, ainsi qu'une possible généralisation aux groupes algébriques commutatifs qui entraînerait ces conjectures.

(a) FORMES LINÉAIRES DE LOGARITHMES.

Soient $a_{1}, \ldots, a_{n} \in \mathbf{N}^{*}, b_{1}, \ldots, b_{n} \in \mathbf{Z}$ et $S$ un ensemble fini de places de $\mathbf{Q}$. On suppose $a_{1}^{b_{1}} \ldots a_{n}^{b_{n}} \neq \pm 1$, on pose $\log N v=\log p$ si $v$ est la place $p$-adique, $\log N v=1$ si $v=\infty$, et

$$
\Sigma=-\sum_{v \in S} \log \left|a_{1}^{b_{1}} \ldots a_{n}^{b_{n}} \mp 1\right|_{v}
$$

Conjecture. Avec les notations ci-dessus on a

$$
\Sigma \leqslant C \mathrm{e}^{c n} \cdot \sum_{v \in S} \log N v \cdot \max _{1 \leqslant i \leqslant n} \log \left(\left|b_{i}\right|+1\right) \cdot \prod_{i=1}^{n}\left(1+\log a_{i}\right)
$$


où $C, c$ sont des constantes absolues.

Cette conjecture revient à regrouper pour plusieurs places les minorations actuellement connues pour chaque place individuellement et le seul point encore non démontré est la forme du facteur $\sum_{v \in S} \log N v$ (une compilation naïve des résultats actuellement disponibles donne $\sum_{v \in S} N v$ à la place de ce facteur. En procédant de même avec les conjectures les plus optimistes formulées par S. Lang, M. Waldschmidt et aliter (voir [5], p. 212) pour la place archimédienne on aboutit à :

Conjecture optimiste. Avec les notations ci-dessus on a

$$
\Sigma \leqslant c\left(\sum_{v \in S} \log N v+\sum_{i=1}^{n} \log \left(\left|b_{i}\right|+1\right)+\sum_{i=1}^{n} \log a_{i}\right)+C
$$

où $C, c$ sont des constantes absolues.

En fait, la conjecture de Lang-Waldschmidt suggère que pour tout $\varepsilon>0$ la conjecture précédente soit valide avec $c=1+\varepsilon$ et $C$ remplacé par $n . C(\varepsilon)$. On s'attend qu'une telle conjecture ait des conséquence fortes, nous allons montrer un exemple plausible à la section suivante.

(b) Application à la CONJECTuRE $a b c$.

PROPOSITION. La conjecture optimiste du paragraphe précédent entraîne la conjecture abc sous la forme suivante: il existe un réel $\kappa>0$ tel que pour tous $x, y, z \in \mathrm{N}^{*}$ satisfaisant $(x, y, z)=1$ et $x+y=z$ on ait $z \leqslant\left(\prod_{p \mid x y z} p\right)^{\kappa}$, où le produit porte sur tous les premiers $p$ divisant $x y z$.

DÉmonstration. Soient $x, y, z \in \mathrm{N}^{*}$ satisfaisant $(x, y, z)=1$ et $x+y=z$, posons $R=\prod_{p \mid x y z} p$. On prend $\left\{a_{1}, \ldots, a_{n}\right\}=\{p \mid x y\}, x / y=a_{1}^{b_{1}} \ldots a_{n}^{b_{n}}$ et $S=\{p \mid z\}$. On a $\log z=-\sum_{p \in S} \log |z / y|_{p}=-\sum_{p \in S} \log \left|a_{1}^{b_{1}} \ldots a_{n}^{b_{n}}+1\right|_{p}$

$$
\sum_{v \in S} \log N v+\sum_{i=1}^{n} \log a_{i}=\log R
$$

Posons $I_{t}=\left\{i ; \mathrm{e}^{t} \leqslant\left|b_{i}\right|+1<\mathrm{e}^{t+1}\right\}$ et notons $\left|I_{t}\right|$ le cardinal de $I_{t}$, on a $\sum_{t \geqslant 0}\left|I_{t}\right|=n \leqslant \log R$ et

$$
2 \log z+\log R \geqslant \log (x y)+n \geqslant \sum_{i=1}^{n}\left(\left|b_{i}\right|+1\right) \geqslant \sum_{t \geqslant 0}\left|I_{t}\right| \cdot e^{t} \geqslant \sum_{t>\delta}\left|I_{t}\right|(t-\delta) \mathrm{e}^{\delta+1}
$$

pour tout $\delta \in \mathrm{N}$, d'où

$$
\begin{aligned}
\sum_{i=1}^{n} \log \left(\left|b_{i}\right|+1\right) & \leqslant \sum_{t \geqslant 0}\left|I_{t}\right| \cdot(t+1) \\
& \leqslant n(\delta+1)+\sum_{t>\delta}\left|I_{t}\right|(t-\delta) \leqslant(\delta+2) \log R+2 \mathrm{e}^{-\delta-1} \cdot \log z .
\end{aligned}
$$


Utilisant la conjecture optimiste on obtient $\log z \leqslant c\left((\delta+3) \log R+2 \mathrm{e}^{-\delta-1} \log z\right)+C$ et en choisissant convenablement $\delta$ (c'est-à-dire $\delta=[\log (4 c)]$ ) il suit

$$
\log z \leqslant 2 c(3+\log (4 c)) \log R+2 C,
$$

c'est-à-dire $z \leqslant \mathrm{e}^{2 C} \cdot\left(\prod_{p \mid x y z} p\right)^{c_{1}}$ où $c_{1}:=2 c(3+\log (4 c))$.

Réciproquement notons $\Sigma_{\max }=-\sum_{v} \min \left(0 ; \log \left|a_{1}^{b_{1}} \ldots a_{n}^{b_{n}} \mp 1\right|_{v}\right)$, c'est effectivement le maximum des sommes $\Sigma$ considérées au paragraphe précédent lorsque $S$ varie (en omettant éventuellement la place archimédienne). Appliquons la conjecture $a b c$ au triplet $(x, y, z)$ avec $x=x^{\prime} /\left(x^{\prime}, y^{\prime}\right), y=y^{\prime} /\left(x^{\prime}, y^{\prime}\right), z=x \mp y$ où $x^{\prime}:=\prod_{i: h_{:}>n} a_{i}^{b_{i}}$ et $y^{\prime}=\prod_{i: b_{i}<0_{i}} a_{i}^{-b_{i}}$, on obtient:

$$
\Sigma_{\max }=\max (\log y ; \log |z|) \leqslant \kappa \cdot \sum_{p \mid x y z} \log p \leqslant \kappa \cdot\left(\sum_{p \in S_{\max }} \log p+\sum_{i=1}^{n} \log a_{i}\right)
$$

où $S_{\max }:=\left\{p ;\left|a_{1}^{b_{1}} \ldots a_{n}^{b_{n}} \mp 1\right|_{p}<1\right\}$. Une propriété remarquable de cette majoration est qu'elle ne dépend pas des $b_{i}$, tandis que lorsque $S$ est réduit à un élément par exemple il est clair que la contribution des $b_{i}$ peut être prépondérante dans nos conjectures du paragraphe précédent. La démonstration de la proposition ci-dessus confirme que dans le cas très particulier de la somme $\Sigma_{\max }$ le terme $\sum_{i=1}^{n} \log \left(\left|b_{i}\right|+1\right)$ est bel et bien absorbé par les autres.

Récemment, Baker [1] a formulé une version raffinée de la conjecture $a b c$. Reprenant les notations de la preuve de la proposition et posant $\omega:=\sum_{p \mid x y} 1$, sa conjecture s'énconce: il existe des réels $\kappa_{1}, \kappa_{2}>0$ tels que pour tout $\varepsilon>0$ on ait $z \leqslant \kappa_{1} \cdot R^{1+\varepsilon} \cdot \varepsilon^{-\kappa_{2} \cdot \omega}$, ou, en optimisant sur $\varepsilon$ :

$$
z \leqslant \kappa_{1} \cdot R \cdot \frac{(\log R)^{\kappa_{2} \cdot \omega}}{\left(\kappa_{2} \cdot \omega\right) !} .
$$

Il introduit également la quantité:

$$
\Xi:=\min (1 ;|\log (x / y)|) \times \prod_{p \mid z} \min \left(1 ; p|\log (x / y)|_{p}\right)
$$

qui se compare comme suit à notre $\Sigma_{\max }$ :

$$
\Sigma_{\max }-\sum_{p \mid z} \log p \leqslant-\log \Xi \leqslant \Sigma_{\max }-\sum_{p \mid z} \log p+\log (e-1) .
$$

Enfin, il montre que sa version raffinée de la conjecture $a b c$ est équivalente à la minoration $\Xi \geqslant \kappa_{3} \cdot \prod_{p \mid x y} p^{-1}\left(\varepsilon^{\kappa_{2} \cdot \omega} \cdot x^{-\varepsilon}\right)^{1 /(1+\varepsilon)}$ pour tout $\varepsilon>0$, mais il manque d'ajouter que le facteur $x^{-\varepsilon}$ est alors minoré par $\kappa_{1}^{-\varepsilon} \cdot R^{-\varepsilon-\varepsilon^{2}} \cdot \varepsilon^{\varepsilon \kappa_{2} \cdot \omega}$ et que la minoration de $\Xi$ ainsi obtenue ne 
dépend en fait pas des $b_{i}$. En tout état de cause, ces minorations de formes linéaires de logarithmes déduites de la conjecture $a b c$ ne s'appliquent qu'aux quantités $\Sigma_{\max }$ et ne fournissent pas d'information signicative sur le cas général de nos conjectures du paragraphe précédent, par exemple.

(c) GÉNÉRALISATION AUX GROUPES ALGÉBRIQUES COMMUTATIFs.

Formulons une conjecture bivariée généralisant le théorème du sous-espace d'une part et les minorations de formes linéaires de logarithmes d'autre part, à un groupe algébrique commutatif quelconque. Le théorème du sous-espace correspondra alors au cas des puissances du groupe additif et on explicitera le cas des puissances du groupe multiplicatif.

Soient $G$ un groupe algébrique commutatif de dimension $g$, défini et plongé dans un espace projectif $\mathbf{P}_{n}$ sur $\overline{\mathbf{Q}}$. On note $h$ la hauteur projective (logarithmique, absolue) des sous-variétés de $\mathbf{P}_{n}$ définies sur $\overline{\mathbf{Q}}, \widehat{h}$ la hauteur normalisée des sous-variétés de $G$ et $H:=$ $\exp h$. Rappelons qu'on appelle sous-variété de torsion de $G$ une sous-variété algébrique qui est translatée d'un sous-groupe algébrique par un point de torsion. Si $K$ est un corps de nombres, on désigne par $K_{v}$ le complété de $K$ en la place $v$ de $K, N v$ la norme de la place $v$ (avec la convention $\log N v=1$ si $v$ est une place archimédienne) et $\widehat{\operatorname{Dist}}_{v}(x, \cdot)$ une distance appropriée d'un point de $x \in \mathbf{P}_{n}\left(K_{v}\right)$ à un translaté de sous-groupe algébrique de $G$. Pour les puissances des groupes additif et multiplicatif on explicitera ci-après cette distance, pour une variété abelienne on pourra prendre la plus petite distance d'un logarithme de $x$ à l'espace tangent du translaté de sous-groupe considéré. Dans le cas d'un produit de tels groupes on formulera une version multiprojective de notre conjecture, mais dans le cas d'une extension non dégénérée d'une variété abelienne par un group linéaire les fonctions $\widehat{h}$ et $\widehat{\operatorname{Dist}}_{v}(x, \cdot)$ restent à définir précisément.

Conjecture. Soient $G$ un groupe algébrique commutatif de dimension $g$, défini et plongé dans un espace projectif $\mathbf{P}_{n}$ sur un corps de nombres $K_{0}$, et $\varepsilon>0$, alors il existe un réel $c(\varepsilon) \geqslant 1$ tel que l'énoncé suivant soit vérifié.

Soient $K$ une extension finie de $K_{0}$ et $S$ un ensemble fini de places de $K$ (contenant les places archimédiennes), on se donne des translatés de sous-groupes algébriques (respectivement sous-variétés de torsion) $T_{1}, \ldots, T_{\ell} \subset G$ définis sur $\overline{\mathbf{Q}}$ et tels que $\bigcap_{i=1}^{\ell} T_{i}=\emptyset$. Alors il existe une famille finie de translatés de sous-groupes algébriques (respectivement sous-variétés de torsion) dont la réunion contient tous les $x \in G(K)$ pour lesquels

$$
\sum_{v \in S} \sum_{i=1}^{\ell} \frac{\left[K_{v}: \mathbf{Q}_{v}\right]}{[K: \mathbf{Q}]} \cdot \log \widehat{\operatorname{Dist}}_{v}\left(x, T_{i}\right)
$$

est majoré par

$$
-\sum_{i=1}^{\ell}\left((1+\varepsilon) \cdot \widehat{h}(x)+\widehat{h}\left(T_{i}\right)+c(\varepsilon) \cdot \log \operatorname{deg}\left(T_{i}\right)+\frac{c(\varepsilon)}{g} \cdot\left(h(\bar{G})+\sum_{v \in S} \log N v\right)\right) .
$$


Cette conjecture, qui peut donc être vue comme un « collage $\gg$, met en regard les énoncés de type théorème du sous-espace (cas des translatés de sous-groupes) et les énoncés de type minoration de formes linéaires de logarithmes (cas des sous-variétés de torsion). Notons que la majoration demandée dans cette conjecture est plus contraignante que celle qui apparait dans la conjecture principale de Vojta [10, Chapter 3.4], (c'est-àdire, on retient moins de points exceptionnels que dans cette référence), en revanche on est plus exigeant sur la nature de l'ensemble algébrique contenant les points exceptionnels et sur la formulation des « constantes $\gg$. Notons également que dans le cas des translatés de sous-groupes cette formulation des « constantes $\gg$ est superflue et ne prendrait de sens qu'en précisant davantage l'ensemble algébrique contenant les points exceptionnels (par exemple, en donnant une borne pour son degré). Nous ne nous risquons pas dans cette direction, mais nous avons gardé la même majoration pour les deux cas afin d'en accentuer le parallélisme.

Lorsque $G$ est une variété abelienne le cas des translatés de sous-variétés abeliennes se déduit essentiellement du théorème 2 de [3]. Explicitons cette conjecture dans les cas des puissances des groupes additif et multiplicatif respectivement.

LE CAS $G=\mathbf{G}_{a}^{n}$ : On a $\mathbf{G}_{a}^{n}(K)=K^{n} \subset \mathbf{P}_{n}(K)$, prenons les translatés de sousgroupes $T_{i}$ de codimension 1, ce sont des hyperplans définis par des formes linéaires $L_{i} \in \overline{\mathbf{Q}}\left[X_{0}, \ldots, X_{n}\right]$ telles que $L_{1}, \ldots, L_{\ell}$ soient sans point commun non trivial (si $\ell=n+1$ ceci est équivalent à demander que $L_{1}, \ldots, L_{n+1}$ sont linéairement indépendantes). On a $\widehat{\operatorname{Dist}}_{v}\left(x, T_{i}\right)=\left|L_{i}(x)\right|_{v} /\left(\left\|L_{i}\right\|_{v} \cdot\|x\|_{v}\right)$ par définition, et la conjecture entraîne que si $x \in \mathrm{G}_{a}^{n}(K)$ satisfait

$$
\prod_{v \in S} \prod_{i=1}^{\ell}\left(\frac{\left|L_{i}(x)\right|_{v}}{\left\|L_{i}\right\|_{v} \cdot\|x\|_{v}}\right)^{\left[K_{v}: \mathbf{Q}_{v}\right] /[K: \mathbf{Q}]}<\frac{1}{H(x)^{\ell(1+\varepsilon)} \cdot \prod_{i} H\left(L_{i}\right) \cdot\left(H\left(\mathbf{P}_{n}\right) \prod_{v \in S} N v\right)^{c(\varepsilon) \ell / n}}
$$

alors $x$ appartient à un nombre fini de sous-variétés linéaires propres de $\mathbf{G}_{a}^{n}$. Lorsque $\ell=n+1$ on retrouve le théorème du sous-espace. On remarque encore que lorsque $G=\mathrm{G}_{a}^{n}$ les hypothèses de la conjecture ne peuvent être satisfaites pour le cas de torsion et, comme nous avons déjà indiqué que la contribution des termes autres que $H(x)$ importe peu dans le cas des translatés de sous-groupes, il suit que lorsque $G=\mathrm{G}_{a}^{n}$ notre conjecture est essentiellement équivalente au théorème du sous-espace.

LE CAS $G=\mathrm{G}_{m}^{n}$. On a $\mathbf{G}_{m}^{n}(K)=\left(K^{*}\right)^{n}$ qu'on considère plongé dans $\mathbf{P}_{1}(K)^{n}$, prenons les translatés de sous-groupes $T_{i}$ de codimension 1 , ils sont définis par des équations de la forme $\prod_{j=1}^{n} X_{j}^{b_{i, j}}-\alpha_{i}=0$ où $b_{i, j} \in \mathrm{Z}$ et $\alpha_{i} \in \overline{\mathbf{Q}}$. Les $\left|b_{i, j}\right|$ sont les multidegrés de $T_{i}$ dans $\mathbf{P}_{1}(K)^{n}$, sa hauteur normalisée est égale à $h\left(\alpha_{i}\right)$ et, pour $x \in \mathbf{G}_{m}^{n}(K)$, on prend

$$
\widehat{\operatorname{Dist}}_{v}\left(x, T_{i}\right) \leqslant\left|\prod_{j=1}^{n} x_{j}^{b_{i, j}}-\alpha_{i}\right|_{v}
$$


Considérons le cas $K=\mathbf{Q}, T_{i}$ défini par $X_{i}-1$ pour $i=1, \ldots, n$ et $T_{n+1}$ défini par $\prod_{j=1}^{n} X_{j}^{b_{j}}-\alpha, \alpha \in \overline{\mathbf{Q}} \backslash\{1\}$ (respectivement $\alpha$ racine de l'unité distincte de 1), alors la conjecture entraîne qu'il existe un nombre fini de translatés de sous-groupes (respectivement de sous-variétés de torsion) de $\mathbf{G}_{m}^{n}$ tels que si $a_{1}, \ldots, a_{n} \in \mathbf{N}^{*}$ et $\left(a_{1}, \ldots, a_{n}\right)$ n'appartient à aucun de ces translatés, on a :

$$
\prod_{v \in S}\left|\prod_{j=1}^{n} a_{j}^{b_{j}}-\alpha\right|_{v} \geqslant \frac{1}{H(\alpha) \cdot \prod_{j=1}^{n}\left(a_{j}^{2+\varepsilon}\left|b_{j}\right| c(\varepsilon)\right) \cdot\left(H\left(\mathbf{P}_{n}^{m}\right) \prod_{v \in S} N v\right)^{c(\varepsilon)(1+1 / n)}} .
$$

En particulier, si $\alpha=-1$ on a $H(\alpha)=1$ et la minoration ci-dessus est valable pour tous $a_{1}, \ldots, a_{n} \in \mathrm{N}^{*}$ multiplicativement indépendants, ce qui correspond à notre conjecture optimiste du (a).

\section{REFERENCES}

[1] A. Baker, 'Linear forms in logarithms and abc-conjecture', in Proc. Conference in Eger (W. de Gruyter, Berlin, 1998).

[2] J.H. Evertse, 'An explicit version of Falting's product theorem and an improvement of Roth's lemma', Acta Arith. 73 (1995), 215-248.

[3] G. Faltings, 'Diophantine approximations on abelian varieties', Ann. of Math. 133 (1991), 549-576.

[4] R. Ferretti, 'An effective version of Falting's product theorem', Forum Math. 8 (1996), 401-427.

[5] S. Lang, Elliptic curves: diophantine analysis (Springer Verlag, Berlin, Heidelberg, New York, 1978).

[6] M. Nakamaye, 'Multiplicity estimates and the product theorem', Bull. Soc. Math. France 123 (1995), 155-188.

[7] P. Philippon, 'Nouveaux lemmes de zéros dans les groupes algébriques commutatifs', Rocky Mountain J. Math. 26 (1996), 1069-1088.

[8] H.P. Schlickewei, 'The p-adic Thue-Siegel-Roth-Schmidt theorem', Arch. Math. 29 (1977), 267-270.

[9] W.M. Schmidt, 'The subspace theorem in diophantine approximation', Compositio Math. 69 (1989), 121-173.

[10] P. Vojta, Diophantine approximations and value distribution theory, Lecture Notes in Math. 1239 (Springer Verlag, Berlin, Heidelberg, New York, 1997).

UMR 9994 du CNRS-Problèmes Diophantiens

Université P. \& M. Curie, T46-56, 5ème ét.

F-75252 Paris Cedex 05

France 